\title{
Breeding soundness evaluation of bulls by semen analysis, testicular fine needle aspiration cytology and trans-scrotal ultrasonography
}

Chapwanya, A., Callanan, J., Larkin, H., Keenan, L. and Vaughan, L. School of Agriculture, Food Science and Veterinary Medicine, Veterinary Medicine Centre, UCD, Belfield, Dublin 4, Ireland

\begin{abstract}
The aim of this study was to evaluate the usefulness of trans-scrotal ultrasonography and testicular fine needle aspiration cytology in assessing bulls for breeding suitability. These two techniques were also compared with semen analysis. Bulls presented for breeding soundness evaluation were assessed using all three techniques. The findings of each technique were compared. There was agreement in classification of fertile bulls using all three techniques, suggesting that the combined use of these techniques enhances routine breeding soundness examination. Use of the three techniques also enhances detailed investigation of suspected sub-fertile bulls while accurately identifying testicular cause(s) of sire sub-fertility.
\end{abstract}

Key Words: breeding soundness, bulls, fine needle aspirate, semen evaluation, ultrasonography

Irish Veterinary Journal

\section{Corresponding author:}

Lindy Vaughan

School of Agriculture, Food Science and Veterinary Medicine,

Veterinary Medicine Centre, UCD, Belfield, Dublin 4, Ireland

Tel: +3531716 6089

Fax: +3531716 6091

Email: lindy.vaughan@ucd.ie

\section{Introduction}

Reproductive soundness evaluation facilitates the elimination of sub-fertile sires from breeding programmes. Although the most conclusive evidence of fertility is made on the basis of pregnancy rate in the females served, breeding soundness evaluation of the male offers predictive information on expected performance that may enhance overall herd productivity. Although the use of artificial insemination (AI) is widespread, natural service is commonly used in suckler cow enterprises and to 'sweep up' following a period of AI in dairy herds.

Clinical examination, observation of mating performance and behaviour and semen evaluation has been traditionally used to assess bulls for suitability for breeding (Parkinson, 2004). Trans-scrotal ultrasonography and testicular fine needle aspiration (FNA) cytology are two additional techniques that can be used to diagnose sub-fertility in bulls (Bagley and Chapman, 2005) and dogs (Dahlbom et al., 1997).

Trans-scrotal scanning allows visualisation of tissue interfaces within the scrotum, as well as the accurate measurement of scrotal circumference (Love, 1992). Transscrotal scanning also allows assessment of both palpable and non-palpable testicular lesions (Ahmad and Noakes, 1995). In addition, the technique is convenient and non-invasive making it ideal for on-farm situations.

Testicular FNA cytology, on the other hand, identifies obstructive and non-obstructive forms of sub-fertility. Additional advantages of the FNA technique are its low cost (Verma et al., 1989) and simplicity.

Successful management of viable cattle enterprises demands implementation of efficient breeding programmes. Identification of fertility problems in beef and dairy herds requires accurate diagnostic techniques for bull sub-fertility in order to arrest declining trends in fertility. This study was designed to evaluate whether testicular FNA cytology and trans-scrotal ultrasonography can be employed in assessing fertility in bulls, and to determine their usefulness in the diagnosis of testicular lesions.

\section{Materials and methods}

Thirty-two bulls aged two to nine years (mean $5.6 \pm 1.6$ yrs) were examined at the UCD University Veterinary Hospital between March and November 2000. The breeds represented were Friesian $(\mathrm{n}=9$, mean age $=5.3 \pm 1.1 \mathrm{yrs})$, Charolais $(\mathrm{n}=6$, mean age $=6.3 \pm 1.1 \mathrm{yrs})$, Hereford $(\mathrm{n}=$ 6 , mean age $=6.5 \pm 1.1 \mathrm{yrs})$, Jersey $(\mathrm{n}=2$, mean age $=7 \pm$ $1.0 \mathrm{yrs})$, Red Dane $(\mathrm{n}=3$, age $=5.7 \pm 0.5 \mathrm{yrs})$, Limousin $(\mathrm{n}$ $=3$, age $=4.5 \pm 1.5 \mathrm{yrs})$, Simmental $(\mathrm{n}=2$, age $=5 \mathrm{yrs})$ and Aberdeen Angus ( $\mathrm{n}=1$, age $=3 \mathrm{yrs}$ ). A detailed history and signalment were obtained for each bull.

Clinical examination was performed with each bull restrained in a crush. External genitalia were examined by visual assessment and palpation for testicular tone. Scrotal 
circumference was measured using a metal scrotal tape (McGowan et al., 1995). Trans-scrotal ultrasonography was performed on both testes while testicular FNA cytology was performed on the right testis. Semen evaluation was also performed for each bull.

A B-mode, real time portable ultrasound scanner with a $7.5 \mathrm{MHz}$ linear array transducer (SonoAce 600V, BCF Technology, Edinburgh, Scotland) was used for transscrotal ultrasonography. The technique used for scanning was similar to that described by Ahmad et al. (1991) in small ruminants. Semen was collected using an electroejaculator ('Spaceage' bull ejaculator, Standard Precision Electronics Inc., California) using a technique similar to that described by McGowan et al. (1995).

Semen was assessed on the basis of volume, colour, consistency, gross and progressive motility, live-to-dead ratio, sperm concentration and morphology.

For testicular FNA, each bull was restrained in a crush. An area of scrotal skin measuring $3 \times 3 \mathrm{~cm}$ in the middle lateral region (furthest away from the raphe) of the right testis was clipped and aseptically prepared. The right testis was selected for aspiration because of ease of access to the lateral testicular surface by a right-handed operator. The testis and scrotum were grasped with one hand so that the scrotal skin tensed over the site to be aspirated. A 21 gauge 2.5 " needle attached to a $5 \mathrm{ml}$ syringe was advanced at right angles to the skin and inserted smoothly into the testis using a method similar to that described by Larkin (1994). When the needle was fully inserted into the testis, suction pressure was applied to aspirate testicular tissue. Each testis was only aspirated once. The aspirate was then expelled onto a clean glass slide and spread using the edge of a second glass slide. Each smear was air-dried, labelled and transported to the laboratory.

In the laboratory, the smears were stained with SpeedyDiff (Clin-Tech Limited, Clacton-on-Sea, England). The number of testicular cells (precursor and mature sperm, Sertoli and Leydig cells) was evaluated at a magnification of 400. Using a grading criterion similar to that described by Johnsen (1970), the bulls were allocated a fertility score. The fertility score, based on the ratio of mature spermatozoa to Sertoli cells, ranged from zero to five ( 0 : no cells; 1: few cells; 2-3: moderate cells; 4-5: many cells). The score for each bull was then correlated with semen evaluation and testicular ultrasonographic findings. The semen parameters of volume, motility, percentage live spermatozoa and percentage morphologically normal spermatozoa were analysed using the ANOVA for different means. The confidence interval was set at $p=0.05$. The results of testicular FNA cytology and semen analysis, semen analysis and testicular ultrasound, testicular FNA cytology and testicular ultrasound were compared using the Chi- square test.

\section{Results}

The average scrotal circumference of the 32 bulls was 31 $\mathrm{cm}$ (range $28-41 \mathrm{~cm}$ ). Twenty-one bulls producing semen with a progressive motility of at least $40 \%$, and no less than
Figure 1: Testicular FNA cytology showing normal proportions of testicular cells from a healthy six-year-old Angus bull. (Magnification x400, Speedy-Diff stain).

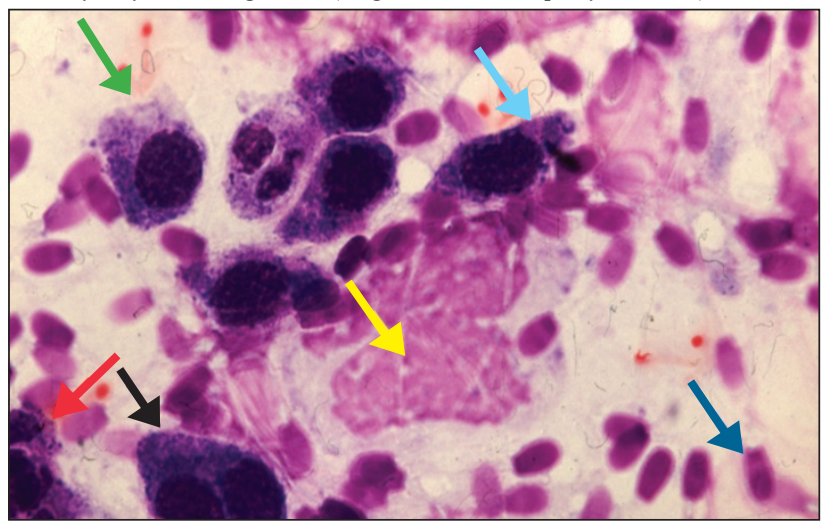

The spermatogenic cells identifiable are:

Spermatogonia $(\longrightarrow)$

Spermatocytes $(\longrightarrow)$

Spermatids $(\longrightarrow)$

Mature spermatozoa $(\longrightarrow)$

Sertoli cells $(\longrightarrow)$.

Some amorphous material $(\longrightarrow)$ is also seen.

$50 \%$ morphologically normal spermatozoa, were identified as suitable for breeding. Eleven bulls produced semen of poor quality and were deemed sub-fertile (fail; Table 1). Testicular aspirates from 24 bulls yielded normal proportions of testicular cells, i.e., a Sertoli: sperm ratio $\geq 1$ with intact precursor sperm, mature sperm, Sertoli and Leydig cells (Figure 1). The FNA cytology of the remaining eight bulls exhibited abnormal testicular cellularity. The abnormalities were: too few cells $(n=3)$;

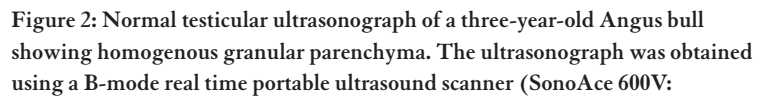
showing homogenous granular parenchyma. The ultrasonograph was obtained using a B-mode real time portable ultrasound scanner (SonoAce $600 \mathrm{~V}$ : Edinburgh) with a $7.5 \mathrm{MHz}$ linear array transducer.
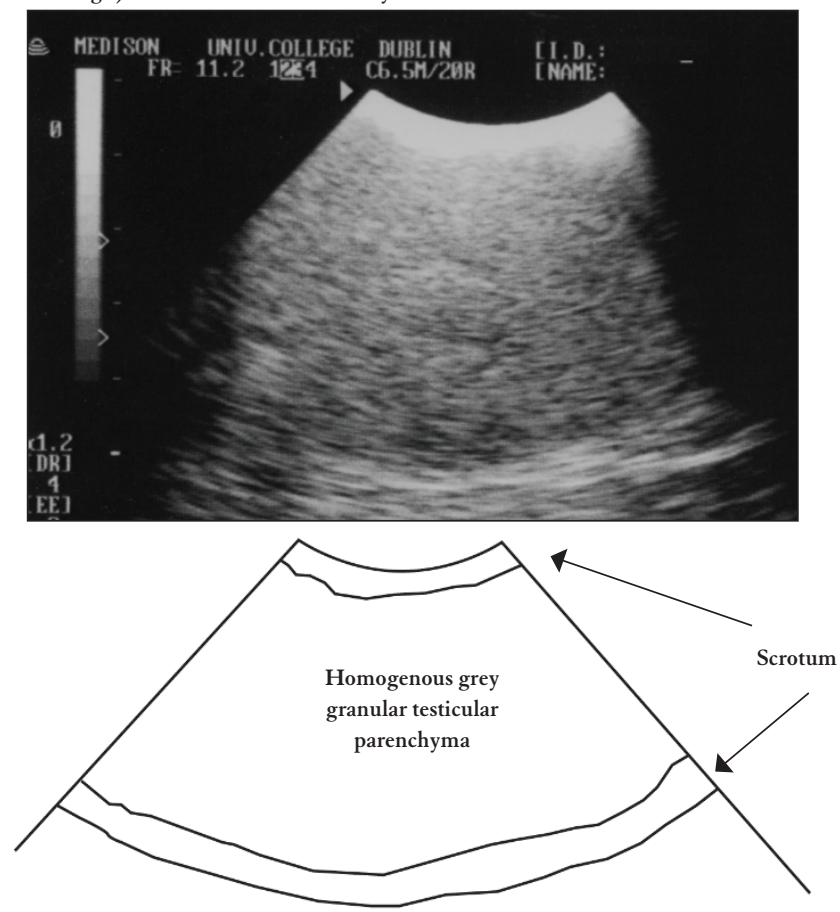
Table 1: Classification of bulls as suitable (pass) or unsuitable (fail) for breeding using semen analysis, testicular FNA cytology and trans-scrotal ultrasonography techniques

Technique

Semen analysis

Testicular FNA cytology

Testicular ultrasonography

fluid aspirated $(n=1)$; only mature sperm aspirated $(n=1)$; and inclusion of white blood cells $(n=3)$.

Twenty-five bulls had no lesions detectable by transscrotal ultrasonography (Figure 2). The remaining seven bulls had abnormal testicular parenchyma and, as such, were considered unsuitable for breeding. The testicular ultrasonographic abnormalities identified were: fluid accumulations $(n=3)$; testicular abscess $(n=1)$; orchitis $(n=1)$; and testicular fibrosis $(\mathrm{n}=2)$. Testicular abseccesation and fibrosis were also identifiable on testicular palpation. Table 1 shows the classification of the 32 bulls using the three techniques as either suitable (pass) or unsuitable (fail) for breeding.

Testicular FNA cytology and semen evaluation together classified 17 bulls as suitable for breeding (Table 2a).

This indicates a significant association between correct classification of bulls on the basis of semen evaluation and on testicular FNA cytology $\left(\chi^{2}=31.32, \mathrm{p}<0.05\right)$. Eleven bulls that were sub-fertile were correctly deemed unsuitable for breeding on the basis of both techniques. Classification of four bulls differed between the two techniques (suitable on FNA cytology while unsuitable on semen analysis). Semen analysis and testicular trans-scrotal ultrasonography together classified 18 bulls as suitable for breeding $\left(\chi^{2}\right.$ $=12.34, \mathrm{p}<0.05)($ Table $2 \mathrm{~b})$. Nine bulls were deemed unsuitable. Five bulls with detectable ultrasonographic abnormalities (fail on ultrasonography) produced semen of acceptable quality (pass on semen analysis).

Table 2: 2x2 Kappa tables showing classification of 32 bulls by semen analysis and testicular FNA cytology (a), semen analysis and testicular ultrasonography (b) and testicular FNA cytology and trans-scrotal ultrasonography (c) as either suitable (pass) or unsuitable (fail) for breeding

$2 \mathrm{a}$

\begin{tabular}{l|l|l|l|}
\multirow{2}{*}{ Technique: FNA } & \multicolumn{4}{|c|}{ Semen analysis } \\
\cline { 2 - 4 } & Pass & Fail & Total \\
\hline Pass & 17 & 4 & 21 \\
\hline Fail & 0 & 11 & 11 \\
\hline Total & 17 & 15 & 32
\end{tabular}

$2 \mathrm{~b}$

\begin{tabular}{|l|l|l|l|}
\multirow{2}{*}{ Technique: U/S } & \multicolumn{3}{|l|}{ Semen analysis } \\
\cline { 2 - 4 } & Pass & Fail & Total \\
\hline Pass & 18 & 5 & 23 \\
\hline Fail & 0 & 9 & 9 \\
\hline Total & 18 & 14 & 32
\end{tabular}

$2 c$

\begin{tabular}{|l|l|l|l|}
\multirow{2}{*}{ Technique: U/S } & \multicolumn{3}{|l|}{ Testicular FNA } \\
\cline { 2 - 4 } & Pass & Fail & Total \\
\hline Pass & 20 & 5 & 25 \\
\hline Fail & 1 & 6 & 7 \\
\hline Total & 21 & 11 & 32
\end{tabular}

U/S - Trans-scrotal ultrasonography. FNA - Testicular FNA cytology
Testicular FNA cytology and trans-scrotal ultrasonography classified 20 bulls as normal and suitable for breeding correctly identifying fertile sires on examination using the two techniques $(\chi 2=14.61, \mathrm{p}<0.05)$ (Table $2 \mathrm{c}$ ).

Trans-scrotal ultrasonography, FNA cytology and semen evaluation together identified 17 bulls as suitable for breeding highlighting the likelihood of correctly identifying fertile bulls as suitable for breeding using the three techniques when performing a breeding soundness evaluation.

\section{Discussion}

The identification of bulls with high potential for fertility by observing their mating behaviour (McGowan et al., 1995), and collection and evaluation of semen is routinely performed. Such procedures, although used in many countries, are not completely satisfactory because they do not allow distinction between high- and medium-fertility bulls.

In this study, 21 bulls identified as suitable for breeding produced semen with a progressive motility of at least $40 \%$ with no less than $50 \%$ morphologically normal spermatozoa. This classification is acceptable to many breed societies (Bierschwal, 1996; Parkinson, 2004), and was applicable to this study, as 19 of the 21 bulls identified as suitable for breeding on the basis of semen evaluation were subsequently mated with cows at a breeding ratio of 1:18 to $1: 50$ in the following breeding season in multiple sire mating systems. The average pregnancy rate for these herds was $61 \%$ (range $52 \%$ to $84 \%$ ) after a breeding season lasting, on average, 10 weeks (range six to 12 weeks).

Seven bulls classified as unsuitable for breeding in this study were eventually culled. Six of these bulls were presented because of a suspicion of sub-fertility. The suspicion was confirmed during this study as the bulls were all deemed unsuitable for breeding. The herds from which four bulls came did not report sub-fertility problems despite the bulls having less than $50 \%$ morphologically normal spermatozoa. This could be explained by the fact that these farms practised multiple-sire mating systems that obviously masked the role of bulls producing poor semen or differences in herd management.

The other bulls deemed unsuitable for breeding on the basis of poor semen quality $(n=4)$ had additional problems of: lameness $(n=1)$; poor body condition $(n=2)$; and squamous cell carcinoma of the third eyelid $(n=1)$.

All spermatogenic cells were identifiable on smears made from normal testes of 21 healthy bulls. In all the normal fertile bulls, it was possible to recover sufficient testicular tissue for evaluation using the FNA technique described. In these fertile bulls, there was close agreement between classification by semen evaluation and testicular FNA cytology. The rest of the bulls $(n=11)$ were sub-fertile, with abnormal testicular cytology. It was common to find disrupted cellular organisation, fragmented nuclei and frayed tubule basement membranes in samples where small quantities of tissue were recovered.

Inadequate samples result in inconclusive smear 
evaluations or provide erroneous diagnosis (Larkin, 1994). When this happens, one has to either obtain another sample or base clinical judgement on the findings of an additional technique such as semen evaluation. In this study, bulls yielding unsuitable FNA samples were re-examined after two weeks, otherwise clinical classification was based on semen evaluation and ultrasonographic findings. The usefulness of trans-scrotal ultrasonography in identifying normal testicular parenchyma has been substantiated in our study. There were no testicular lesions detected in 25 bulls, 21 of which were healthy and fertile. The technique is safe, non-invasive, and reliable and can be performed with the standard $5 \mathrm{Mhz}$ or $7.5 \mathrm{MHz}$ trans-rectal transducer currently used for reproductive evaluation in cows and mares. The assessment of testicular echogenecity allows the diagnosis of both focal and diffuse testicular lesions. Seven bulls in this study had palpable and non-palpable testicular lesions identified by ultrasound scanning, indicating that testicular ultrasonography is a more sensitive and more reliable diagnostic modality than palpation alone. Similar lesions have also been accurately identified in studies on dogs (Pugh et al., 1990).

\section{Conclusions}

Combining the techniques of testicular FNA cytology, trans-scrotal ultrasonography and semen analysis resulted in the accurate identification of fertile bulls while correctly diagnosing testicular causes of bull sub-fertility. This suggests that the use of these techniques together enhances breeding soundness examination in bulls by distinguishing highly fertile from bulls of low fertility.

\section{Acknowledgements}

The authors would like to thank Paul Stanley, Eamonn McNally and Aidan Kelly for technical assistance. The study was supported by an EU Fellowhip and subject of a Master's degree thesis.

\section{References}

Ahmad, N. and Noakes, D.E. (1995). A clinical and ultrasonographic study of induced testicular and epididymal lesions in goats and a ram. Animal Reproduction Science 39: 35-48.

Ahmad, N., Noakes, D.E. and Subandro, A.L. (1991). B-model real time ultrasonographic imaging of testes and epididymis of sheep and goats. Veterinary Record 128: 491-496.

Bagley, C.V. and Chapman, C.K. (2005). Breeding Soundness Evaluation of Bulls Animal Health Utah State University 1: $1-2$.

Bierschwal, C.J. (1996). Revised breeding soundness procedures.

Proceedings of Society of Theriogenology Annual General Meeting. Lexington. pp128-130.

Dahlbom, M., Andersson, M., Juga, J. and Alanko, M. (1997). Fertility parameters in male Irish wolfhounds: a two-year follow-up study. Journal Small Animal Practice 38 (12): 547-550.

Johnsen, S.G. (1970). Testicular biopsy score count. A method for registration of spermatogenesis in human testes: normal values and results in 335 hypogonadal males. Hormones 1: 2-9.

Larkin, H.A. (1994). Veterinary cytology - Fine needle aspiration of masses or swellings on animals. Irish Veterinary Journal 47: 65-73.
Love, C.C. (1992). Ultrasonographic evaluation of the testis, epididymis, and spermatic cord of the stallion. Veterinary Clinics of North America: Equine Practice 8 (1): 167-182.

McGowan, M., Galloway, D., Taylor, E., Entwistle, K. and Johnson, P. (1995). The veterinary examination of bulls. Australian Association of Cattle Veterinarians 9: 21-22.

Parkinson, T.J. (2004). Evaluation of fertility in natural service bulls. The Veterinary Journal 168: 215-229.

Pugh, C.R., Konde, L.J. and Park, R.D. (1990). Testicular ultrasound in the normal dog. Veterinary Radiology 31: 195-199.

Verma, A.K., Ram, T.R. and Kapila, K. (1989). FNA value in the diagnosis of testicular neoplasms. Acta Cytologica 33: 631-634. 